Biol. Cybern. 62, 519-528 (1990)

\title{
On the Cost Functions for the Control of the Human Arm Movement
}

\author{
H. Cruse, E. Wischmeyer, M. Brüwer, P. Brockfeld, and A. Dress \\ Fakultät für Biologie und Fakultät für Mathematik der Universität, Postfach 8640, D-4800 Bielefeld 1, \\ Federal Republic of Germany
}

\begin{abstract}
The aim of our investigation is to understand the mechanisms which control the movement of the human arm. The arm is here considered as a redundant system: the shoulder, elbow and wrist joints, which provide three degrees of freedom, combine to move the hand in a horizontal plane, i.e. a two dimensional space. Thus the system has one extra degree of freedom. Earlier investigations of the static situation led to the hypothesis that independent cost functions were attached to each of the three joints and that the configuration chosen for a given target position is that which provides the minimum total cost (Cruse 1986). The aim of the current investigation was to look for measurable values corresponding to the hypothetical cost functions. Experiments using pointers of different lengths attached to the hand showed that the strategy in choosing the joint angles are independent of the limb length. The muscle force necessary to reach a given angle is increased by a spring mounted across a joint. In this situation the angles of the loaded joint are changed for a given target point to give way to the force effect. This leads to the conclusion that the hypothetical cost functions are not independent of the physiological costs necessary to hold the joint at a given angle. The cost functions seem to depend on joint angle and on the force which is necessary to hold the joint in a given position. Cost functions are measured by psychophysical methods. The results show $U$-shaped curves which can be approximated by parabolas. The position of minimum cost (maximum comfort) for one joint showed no or weak dependency on the angles of the other joints. For each subject these "psychophysical" cost functions are compared with the hypothetical cost functions. The comparison showed reasonable agreement. This supports the assumption that the psychophysically measured "comfort functions" provide a measure for the hypothetical cost functions postulated to explain the targeting movements. Targeting experiments using a four joint arm which has
\end{abstract}

two extra degrees of freedom showed a much larger scatter compared to the three joint arm. Nevertheless, the results still conform to the hypothesis that also in this case the minimum cost principle is applied to solve the redundancy problem. As the cost function for the whole arm shows a large minimum valley, quite a large range of arm positions is possible of about equal total costs. The scatter does not result from pure randomness but seems to be mainly produced by the fact that the angles at the end of the movement depend on the value of the joint angles at the beginning of the movement.

\section{Introduction}

Redundancy is a property often found in biological systems. In such systems the problem occurs of how the redundant system is controlled. This question is addressed here with the example of controlling the movement of the human arm. Redundancy is provided as more joints are free to move than are used for a given task. Thus a given point in the workspace of the arm can be reached by a number of different combinations of joint angles of the arm. The question arises of how the control system selects one of the infinite number of possible positions when trying to reach a given point. In an earlier paper considering the control of the human arm the following hypothesis was proposed (Cruse 1986). To each joint a cost function is attached which defines a cost value for each joint angle. The cost functions show a minimum at about the middle of the angle range of the joint and the cost values increase to either of the extreme angles. The total cost of an arm position is described as the sum of the actual cost values of all joints. When reaching to a given point in the workspace, according to this hypothesis, that arm position is selected out of the geometrically possible 
positions which shows the minimum total cost value. In this way the number of degrees of freedom of the system is reduced and thus the redundancy problem can be solved. The above investigation was restricted to a consideration of the static situation, i.e. only the position of the arm at the endpoint of the movement was examined. In a later investigation (Cruse and Brüwer 1987; Cruse 1989) this minimum cost principle was also successfully applied as an essential part of the hypothesis describing the movement of the arm.

The aim of the current investigation is to examine whether a biological substrate can be found which corresponds to the hypothetical cost functions. For this purpose targeting experiments in which the subject was asked to move the arm from a given start point to a given end point (Cruse 1986) are repeated after changing the geometrical configuration of the arm or loading the arm in such a way that different forces have to be developed to perform the movements. Furthermore psychophysical investigations were carried out to find a subjective measure for the comfort of a given joint angle. The "comfort function" obtained in this way will be compared with the hypothetical cost functions as calculated from the results obtained by the targeting movements.

The results show that the cost functions which can directly be measured by psychophysical experiments seem to be quite similar to the hypothetical cost functions postulated to explain the control of the movement of the human arm. Furthermore the latter seem to depend on the actual physiological costs necessary to hold a given joint position. Finally it will be tested whether the minimum cost principle can also be applied to the four joint arm.

\section{Methods}

As described in two earlier papers (Cruse 1986, Cruse and Brüwer 1987), the movement of the arm is restricted to the horizontal plane and the three joints at shoulder, elbow and wrist are free to move. To measure the values of the joint angles the arm of the subject was laid on an artificial arm having three joints which was free to glide over a horizontal plane. The subjects arm could be clamped to the artificial arm so that the axes of rotation of the corresponding joints of both the human and the artificial arm lay on the same axes. The part corresponding to the hand is prolonged by a pointer with a length of $28 \mathrm{~cm}$ and thus has about the same length as the upper and the lower arm. The joints of the artificial arm carried potentiometers to measure the values of the joint angles. These values could be read via $A-D$ converters directly into the computer (Apple II). As in Cruse and Brüwer (1987), for wrist and elbow joint the zero value corresponds to the position
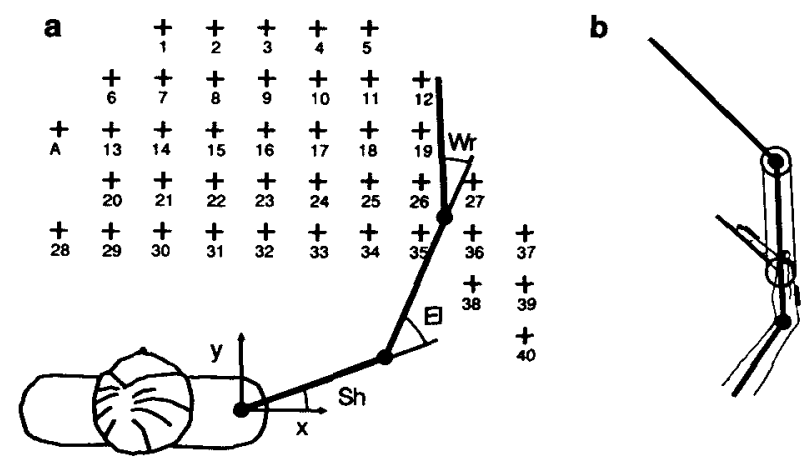

Fig. 1. a Top view of the experimental arrangement. The 41 target points are placed on a horizontal plane in front of the subject. The three measured angles are marked. b A schematical drawing of the distal limbs of the four joint manipulandum. The most distal joint is moved by the fingers in proportion to the angle between fingers and palm. This movement is transduced by means of a wire and two pulleys to the distal joint

when both adjacent limbs are held collinearly. Positive angle values correspond to flexion of the joint. For the shoulder joint the zero position corresponds to the upper arm being held on the prolongation of the line connecting both shoulder joints. Anterior movement is positive. 41 target points are marked on the horizontal plane as shown in Fig. 1a with a regular grid of $10 \mathrm{~cm}$ distance between the points. In the targeting experiments the subject was asked to move the tip of the pointer from a starting point $A$ to each of the target points in a randomized order.

Before the experiment began the subject was asked to perform a series of movements between all target points to become familiar with the situation. During the experiment the subject was asked to make the movements in a "comfortable" way. In particular there was no special request concerning the speed of the movement.

In preliminary experiments two psychophysical methods were tested to obtain a measure for the subjective comfort of a given joint angle. In both cases the subject was first asked to find that position which is most comfortable. This is called the minimum position. This minimum was given the value 1 . Then in the first method the joint under view was moved by the experimenter to a defined angle value and the subject was asked to give by own choice a number which corresponds to the subjective feeling of discomfort (magnitude estimation, Stevens 1958). In a second method the subject was asked to move the joint to a position of a given subjective comfort value and the corresponding joint angle was measured. A comparison showed that both methods gave indistinguishable results. Therefore only the first method was applied for all subjects. 
As the scaling was left completely open to the subjects, quite different numbers were obtained for each subject. For easier comparison the data were later normalized by shifting the minimum value to zero and by multiplying all values of a given subject by one subject-specific factor so that the values of all subjects fall in about the same range. In one case a third method was applied as a form of control. The subject was asked to choose a deliberate joint position, the angle of which was measured. Then the subject had to look for that position beyond the minimum position which possesses the same subjective cost value. These results again fall in the same range as the first two methods.

In another series of experiments the manipulandum was changed to investigate an arm having four joints and thus two extra degrees of freedom. For this purpose the pointer attached to the hand was replaced by two limbs of the same length, $28 \mathrm{~cm}$ each which were connected by a joint carrying another potentiometer connected to the A-D converter. This joint could be moved by the fingers by means of a lever arm and a pulley (Fig. 1b).

\section{Results}

\subsection{Influences of Limb Length on the Cost Functions}

Earlier investigations of the static situation led to the hypothesis that independent cost functions were attached to each of the three joints and that the configuration chosen for a given target position is that which provides the minimum total cost. The existence of these cost functions cannot be shown directly. However, there is an indirect method. It can be tested whether the choice of the arm position for reaching a given target point depends only on the values of the joint angles as proposed by the minimum cost principle or whether it also depends on other factors such as, for example, the length of a limb. In other words, the question is whether the cost functions only depend on the joint angles and not on other properties of the arm geometry. To test this the subjects were investigated by elongating the most distal limb using two different lengths of the pointer attached to the hand. To be able to directly compare the results of the different experiments the following procedure was applied. The subject performed targeting movements to 15 target points (see below) using the usual pointer of a length of $28 \mathrm{~cm}$. In the second session the pointer was prolonged to measure $56 \mathrm{~cm}$ and 15 new target point were given. These new target points were selected separately for each subject in the following way. For the arm position of each original target point the hand was prolonged by $28 \mathrm{~cm}$ and the end of this line was used as new target point. Using this method, the angles of the positions of

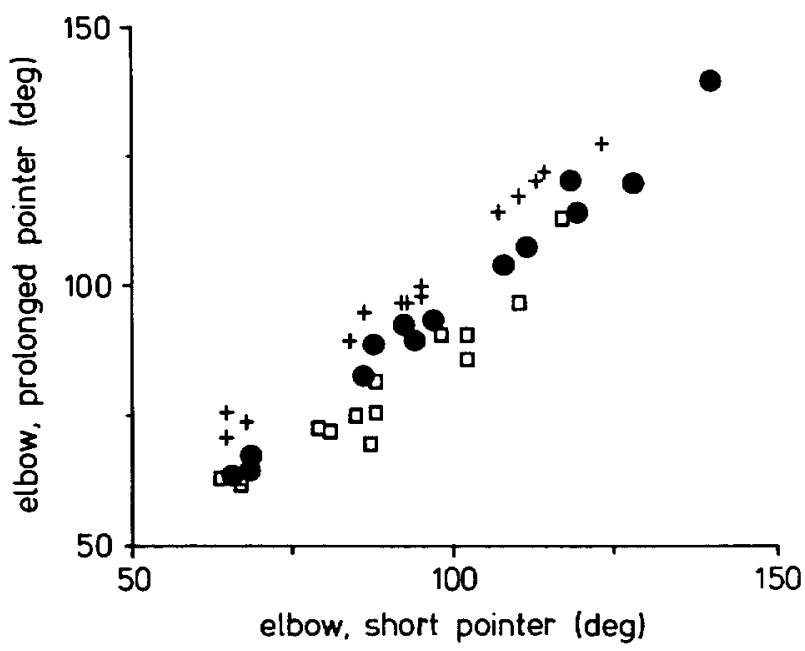

Fig. 2. Comparison of the elbow angles when the distal limb of the three joint arm is short $(28 \mathrm{~cm})$ or long $(56 \mathrm{~cm})$. The target points for the short and the long pointer are selected so that the same angle value should appear when only the angle values but not the limb length influenced the arm position. The three symbols represent the results of three different subjects. Each symbol shows the results of two individual measurements

each pair of original and new target points (the latter with the prolonged pointer) should be equal if the change of the limbs length does not influence the form of the cost functions.

The results are shown for three subjects in Fig. 2. Each dot shows the value of the elbow angle in the original situation (abscissa) and the situation with the prolonged pointer (ordinate). 15 target points were used for each subject. These were points No. 1, 2, 3, 6, 8, 10, 13, 15, 18, 20, 24, 26, 30, 34, and 36 (Fig. 1a). Thus, the points are distributed over nearly the whole range of the workspace investigated here. The slope of the line of minimal squared distances amounts to 0.92 , 0.94 , and 0.99 for the three subjects (corr. coeff. are 0.91, 0.93 , and 0.95 , respectively). This shows that no significant effect from the length of the distal limb can be found on the values of the selected angles although the geometry of the whole arm was changed considerably.

\subsection{Dependency of Joint Angles on External Load}

In the following series of experiments targeting movements are performed when a joint was subject to an additional external load. The joint angles adopted at the target point are measured. The loading was done in two ways.

First, the lower arm was loaded by a constant weight of 500,1000 , or $1500 \mathrm{~g}$ so that this force was pulling at about the middle of the length of the lower arm and in the direction perpendicular to its long axis. The comparison of the elbow angles in the differently 
Table 1. Mean deviation of elbow angle (degrees \pm S.D.) compared to the unloaded situation. $n=20$

\begin{tabular}{lrrr}
\hline Subject & \multicolumn{1}{c}{$500 \mathrm{gr}$} & $1000 \mathrm{gr}$ & \multicolumn{1}{c}{$1500 \mathrm{gr}$} \\
\hline 1 & $7.1 \pm 3.5$ & $7.3 \pm 5.0$ & $7.2 \pm 6.5$ \\
2 & $-1.5 \pm 7.1$ & $-3.1 \pm 6.6$ & $0.5 \pm 7.9$ \\
3 & $-0.2 \pm 7.6$ & $4.4 \pm 7.3$ & $6.0 \pm 5.8$ \\
4 & $6.2 \pm 2.3$ & $7.3 \pm 3.0$ & $3.8 \pm 3.4$ \\
5 & $2.0 \pm 2.2$ & $0.8 \pm 1.8$ & $1.9 \pm 2.5$ \\
6 & $-0.2 \pm 1.9$ & $-0.1 \pm 1.5$ & $-0.2 \pm 1.9$ \\
7 & $7.1 \pm 5.9$ & $7.9 \pm 5.6$ & $12.9 \pm 4.2$ \\
8 & $7.4 \pm 3.4$ & $8.2 \pm 3.2$ & $9.7 \pm 3.9$ \\
9 & $5.9 \pm 3.1$ & $6.5 \pm 3.5$ & $6.5 \pm 2.9$ \\
\hline
\end{tabular}

Table 2. Mean deviation of elbow angle (degrees) when loaded by a spring $(n=20)$. All deviations are significant $(p<0.01) . r$ is the correlation coefficient between the absolute elbow angle in the unloaded situation for each target point and the deviation when being loaded. Only in one case (subject 3) $r$ is significant different from zero. Subjects 1 to 4 correspond to subjects 1 to 4 of Table 1

\begin{tabular}{|c|c|c|c|}
\hline Subject & $\begin{array}{l}\text { Mean } \\
\pm \text { S.D. }\end{array}$ & $\begin{array}{l}\text { Corr. coeff. } \\
r\end{array}$ & Slope \\
\hline 1 & $9.7 \pm 3.6$ & -0.46 & -0.06 \\
\hline 2 & $7.4 \pm 5.1$ & 0.35 & 0.08 \\
\hline 3 & $15.3 \pm 7.0$ & 0.8 & 0.23 \\
\hline 4 & $8.4 \pm 6.2$ & -0.45 & -0.11 \\
\hline 5 & $8.6 \pm 3.2$ & -0.003 & 0.00 \\
\hline 6 & $7.4 \pm 3.2$ & -0.22 & -0.03 \\
\hline 7 & $8.9 \pm 5.9$ & -0.4 & -0.15 \\
\hline 8 & $13.2 \pm 9.7$ & 0.28 & 0.12 \\
\hline
\end{tabular}

loaded with the unloaded situation for 8 subjects and 20 target points for each gave unclear results. Three subjects showed a significant change of the elbow angle in the direction of the pulling weight for at least one weight value. For the other five subjects could in no case a significant effect be found (Table 1).

The result was different when the second method was applied. Here the value of the additional force depended on the value of the elbow angle. This was received by mounting a spring across the elbow joint. The force increased approximately linearly from zero at an elbow joint of $125 \mathrm{deg}$ to $2300 \mathrm{~g}$ at an elbow angle of $30 \mathrm{deg}$. In this case all eight subjects showed a highly significant deviation of the elbow angle in the direction of the pulling force. In only one case was a significant correlation $(p<0.01)$ found between the absolute elbow angle and the deviation (Table 2).

This experiment cannot be sensibly repeated for the shoulder joint when the wrist angle of this subject is about zero. For geometrical reasons in this case no measurable change of the shoulder angle is possible.
Only in one subject was the wrist angle on average large enough to perform this experiment with possible success. In this case also a significant deviation $(1.38 \pm 1.1 \mathrm{deg}, n=20, r=0.19$, slope $=0.007)$ was found.

\subsection{Psychophysical Experiments}

The experiments described above were designed to show properties of the hypothetical cost functions. However, a measurement of the cost function itself was not possible. Psychophysical methods allow the direct measurement of a kind of cost function: the subjects are asked to give a subjective measure for the comfort of a given joint angle. These psychophysical cost functions will be compared later (see Sect. 3.4) with the hypothetical cost functions calculated from the data of the targeting experiments.

As mentioned in the Methods, two ways of measuring the psychophysical cost functions brought the same results. Five subjects $(a$ to $e$ ) were investigated. The results are shown in Fig. 3. They show $U$-shaped curves which can be approximated by parabolas. In general, the two branches of the parabola have different slope values. For the elbow joints these parabolas are shown in Fig. 3. In some cases a function with an exponent greater than two might provide an even better approximation, which was however not investigated in detail.

These experiments were performed by holding the other two joints which were not investigated at a fixed angle. It might be possible that the cost function of one joint might depend on the angle value of another joint. This might in particular be the case for shoulder and elbow joint, as two important muscles, the triceps brachii and the biceps brachii, have tendons which span both joints. The existence of such influences was tested in the following way. The subject was asked to find the minimum position (position of maximum comfort) for the wrist angle for different elbow angles as independent variable. This experiment was repeated using first the wrist angle, then the elbow and finally the shoulder angle as independent variable. The results from 12 subjects are shown in Fig. $4 a-d$. For the results of Fig. $4 \mathrm{a}-\mathrm{c}$ a weak but significant $(p<1 \%)$ correlation was found. For the results of Fig. $4 \mathrm{~d}$ a linear correlation was not calculated because the relation appears to be $U$-shaped. This property was more obvious for 4 individuals. Another subject showed a negative correlation in the experiment shown in Fig. 4b. In general, however, a positive correlation was found. This means that when one joint is flexed, the most comfortable position of the neighbouring joint also is shifted towards increased flexion. 

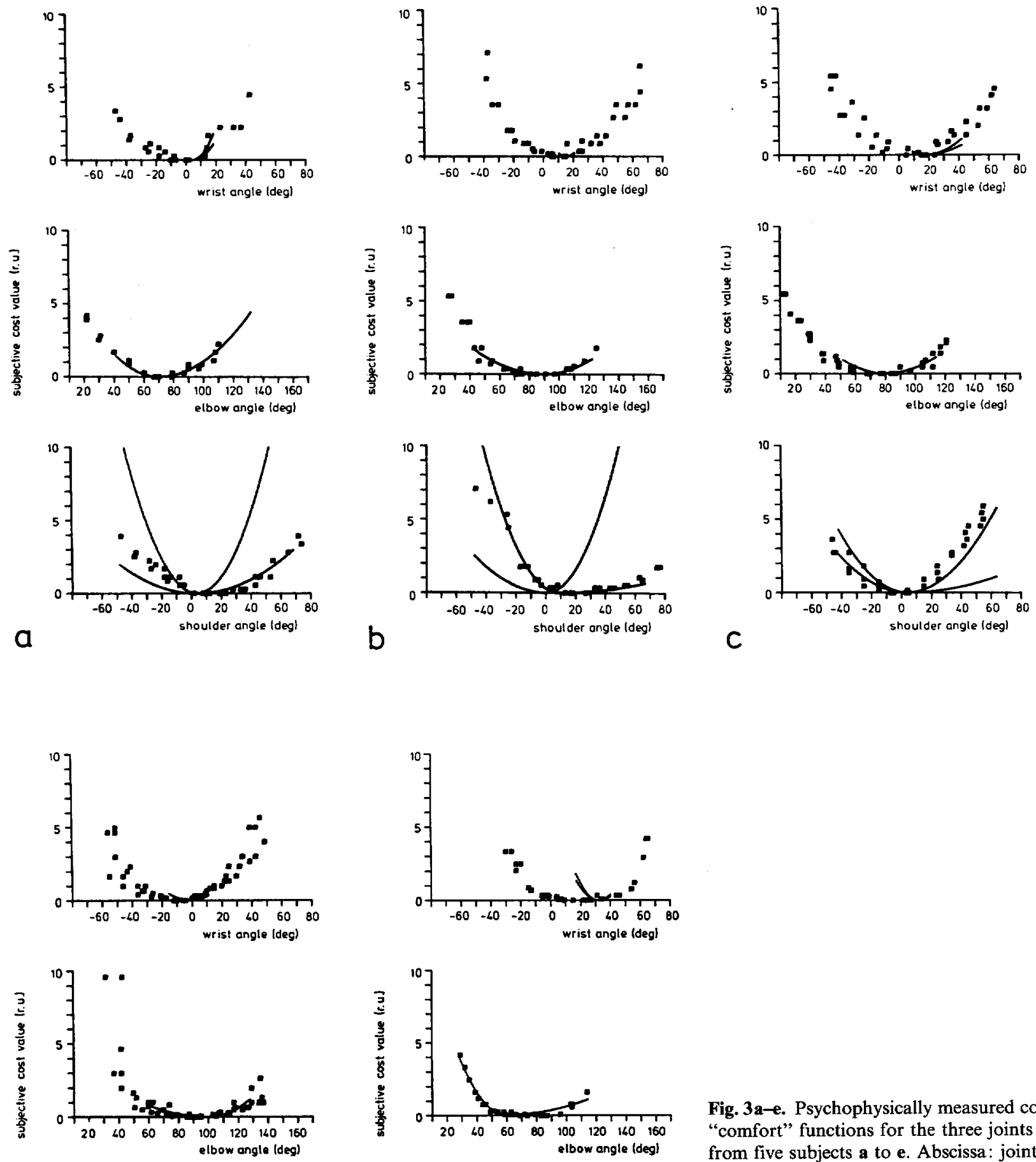

Fig. 3a-e. Psychophysically measured cost or "comfort" functions for the three joints from five subjects a to e. Abscissa: joint angle in degrees. Ordinate: values of
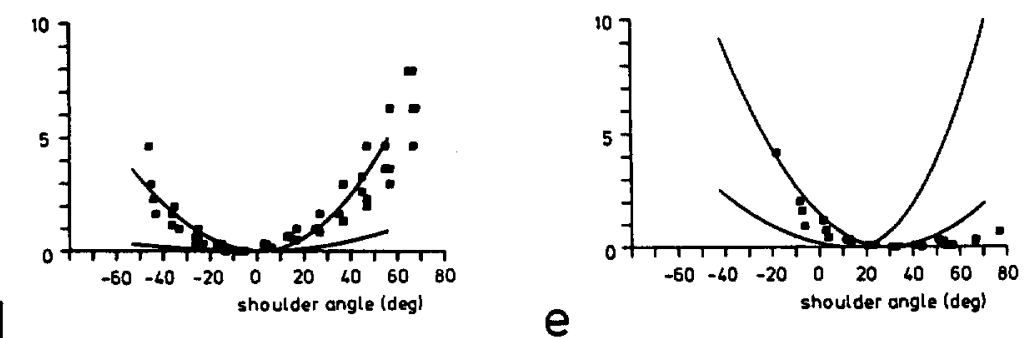
subjective discomfort in relative units. For the elbow those parabola branches are shown which give the best approximation to these values. For shoulder and wrist joint the \pm S.D. range of those parabola branches is shown which are obtained when approximating the targeting experiments 

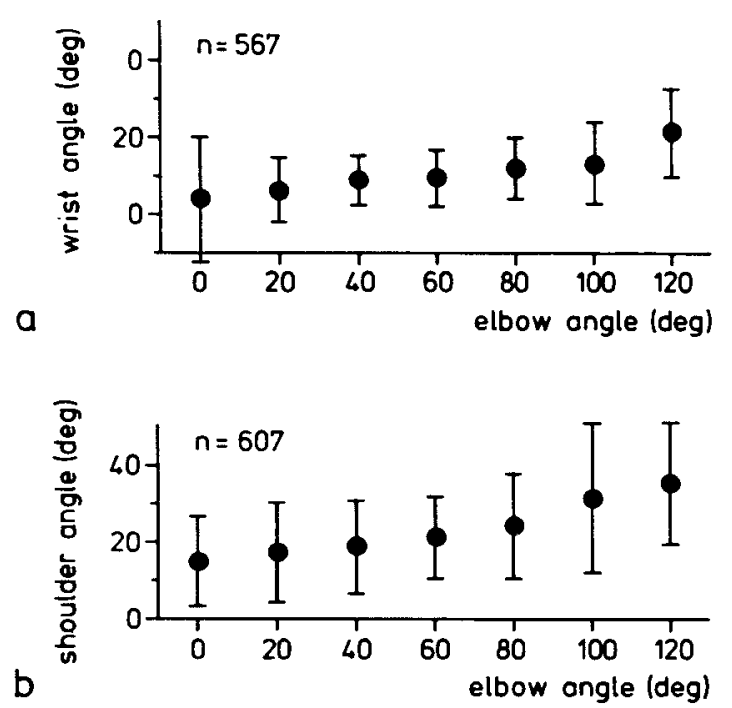
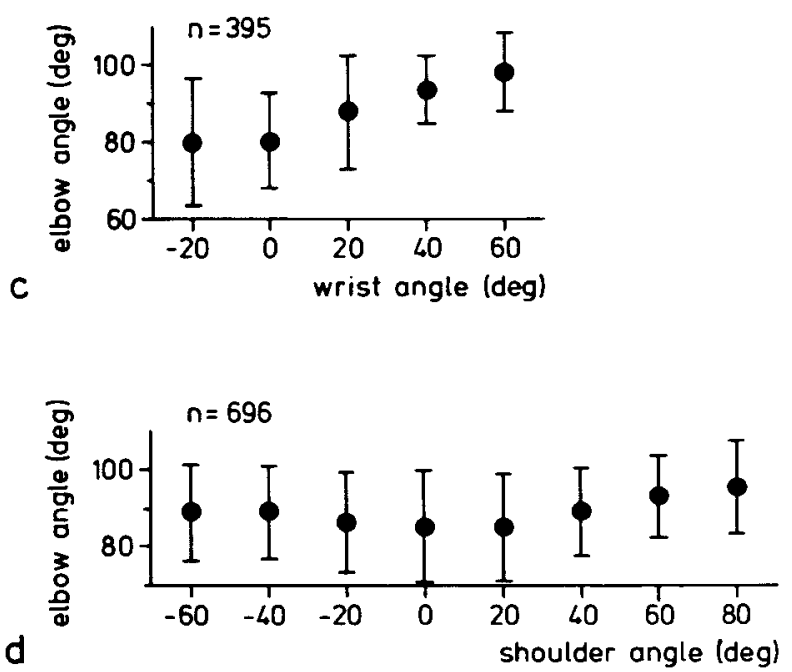

Fig. 4a-d. The angle value of the minimum position of the subjective cost function versus the angle value of another joint (mean value and \pm S.D., 12 subjects). a position of wrist minimum and $\mathbf{b}$ position of shoulder minimum versus the value of the elbow angle. $\mathbf{c}$ position of elbow minimum versus the value of wrist angle. d position of elbow minimum versus the value of the shoulder angle

\subsection{Comparison of Psychophysical and Calculated Hypothetical Cost Functions}

The psychophysical investigations described above revealed nicely $U$-shaped cost functions describing the comfort of the different joint angles. Nevertheless, it is completely open whether these functions are correlated with the hypothetical cost functions postulated in the earlier paper. To investigate this question, the hypothetical cost functions of the five subjects, the psychophysical results of which are shown in Fig. 3, are calculated in the following way. Each subject had to perform targeting movements to 40 (in two cases 41) target points. Each experiment was repeated three times and that angle triple with the median values was taken for further treatment. Then for each subject a triple of cost functions was calculated which could describe these results in the sense of the smallest mean square deviation. This was obtained by an approximation procedure using simulated annealing. Cost functions which consist of parabolas the two branches of which could have different slopes, need three parameters, two for the slope of the two branches and one for the angle value of the minimum. Preliminary calculations showed that allowing all 9 parameters free (three for each of the three cost functions) provides a large scatter in the parameter values of the cost functions whereby some parameters are correlated pairwise. This procedure, therefore, contains superfluous degrees of freedom. To reduce these in order to obtain a more unique result, the procedure was repeated by fixing the parameter of the elbow cost function to those values which were obtained by the psychophysical cost functions. Thus only 6 parameters were free. Still the calculation showed some scatter for the same quality of approximation. When measuring this quality as the mean standard deviation for a joint (mean over all joints and all target positions) these values were $\pm 4.1,6.3,4.0,10.2$, and 5.4 degrees for the subjects $a$ to $e$, respectively. The corresponding mean standard deviation in the targeting experiments was typically \pm 2.9 degrees. To obtain a measure for the mean value and the standard deviation of these 6 parameters for each subject these approximation calculations were repeated ten times (in one case 20 times). In Fig. 3 the psychophysical cost functions of shoulder and wrist joint are compared with the results of the approximation. The latter are shown by those parabola branches which represent the range of \pm S.D. of the cost function obtained by the approximation procedure and for that range of angles which is actually used in the targeting experiment by the corresponding subject. Inspection of Fig. 3 allows a visual comparison between the hypothetical cost function obtained by this approximation procedure and those cost functions obtained by psychophysical experiments. Although not all data points of the psychophysical investigation lie within the range of \pm S.D. of the calculated cost function, the results in general show a reasonable agreement.

\subsection{Four Joint Arm}

Application of the minimum cost principle revealed a sufficient description of the experimental results for the three joint arm having one superfluous degree of freedom. Therefore it was tested whether this method is also applicable when the arm has four joints, i.e. two 
superfluous degrees of freedom. For this purpose the manipulandum was changed to possess four joints and four limbs of about equal length as described in the Methods.

In a preliminary experiment at first the distal joint, i.e. the finger joint, was held fixed at zero angle. Thus the manipulandum corresponded to a three joint arm with a hand pointer of double length as was used in the experiment described in Sect. 3.1. Three subjects performed targeting movements to 15 target points. Then it was tested whether the results could still be described by the minimum cost principle. For the three subjects a sufficient description was found for the "proximal" three joint arm. In addition a corresponding experiment was performed with five subjects by fixing the shoulder angle at zero and investigating the "distal" three joint arm. Here the elbow, the wrist and the remote finger joint were free to move. Also for the 15 target points (which because of the different geometrical situation had to be different to those used in the above experiment) a sufficient approximation was found for the five subjects.

These results encouraged us to apply the hypothesis of the minimum cost principle also to the four joint arm with two degrees of freedom. With these five subjects the usual targeting experiments were performed with the four joint arm using 16 target points. Then an attempt was made to approximate the results by finding four cost functions which could be used to describe the experiments. However, although the distal and the proximal three joint arm alone allowed sufficient approximation, in the four joint arm several approaches provided no adequate results.

Because of this disappointing result we tested by means of a model calculation how in the case of the four joint arm the form of the minimum of the summed cost functions for a given target point looks like. This was done in the following way. A model arm with four cost functions similar to those found in our calculations was used and the sum of the cost functions for a given target point was calculated. In the case of the three joint arm the position of the whole arm and therefore the total cost value is determined when the angle value of one of the three joints is given. Therefore in this case the cost sum could be plotted versus the angle of one joint, e.g. the shoulder joint. In the case of the four joint arm the position of the whole arm is determined only when the angles of two joints are given. Therefore plotting the total cost value for a given target position requires a three-dimensional plot where two joint angles, for example shoulder and elbow angle, are the independent variables. The result of such a calculation showed that the minimum of the total cost function has the form of a valley with quite steep walls. This valley can be very long, reaching over
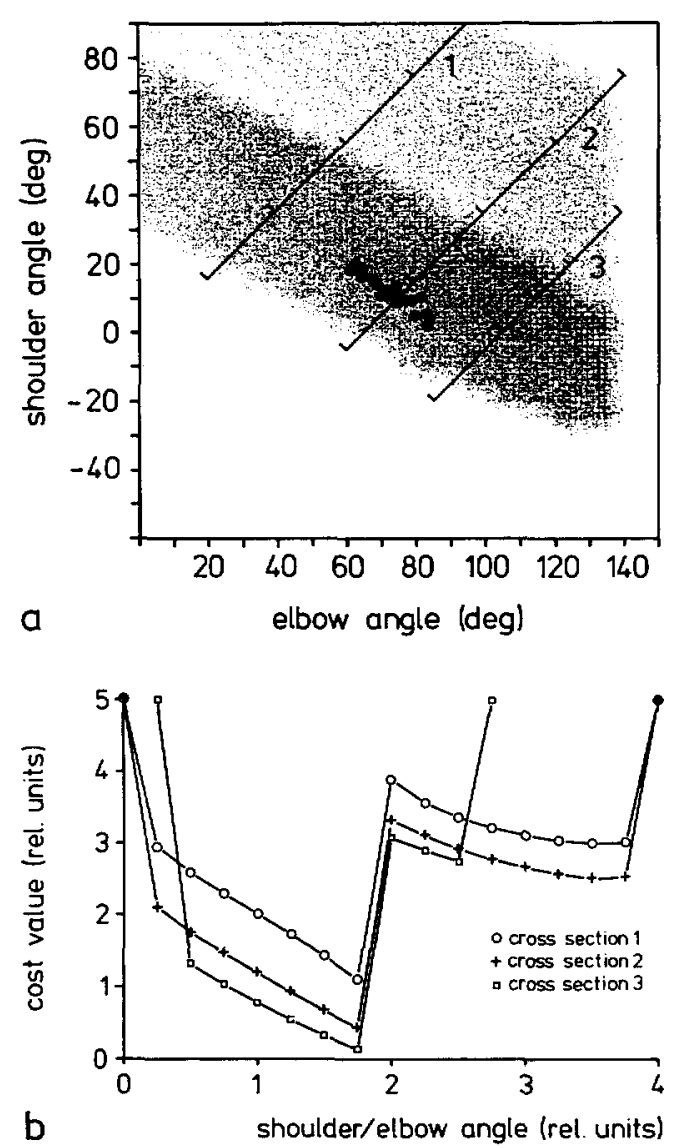

Fig. 5a and b. Presentation of the calculated total cost function of the four joint arm model for a given target point. The form of the four cost functions for the four joints are taken from the approximation results of one subject investigated. a Coordinates are the angles of elbow and shoulder joint. The valley of the cost function is visualized by the density of dots: High density means low total cost values. The dot-free areas represent geometrically impossible arm positions. The filled circles show the experimental results of 25 targeting experiments which each began at a different starting point. $\mathbf{b}$ Three cross sections through the valley along the lines marked in a. Abscissa: combination of angle values as shown in a. Ordinate: Total cost value (relative units)

a large range of the geometrically possible angle values. This is shown for the case of one particular target point in Fig. 5a where the cost values are symbolized by the density of the dots. High density means low costs. The completely dot-free sections show geometrically impossible regions. Three cross sections (Fig. 5b) show the profiles of the cost functions along the corresponding lines in Fig. 5a. The occurrence of such valleys means that for the four joint arm many arm configurations exist which have nearly the same total cost value for a given target point.

Thus even when the control system uses the minimum cost principle, a large scatter is to be expected regarding the arm positions for a given target 


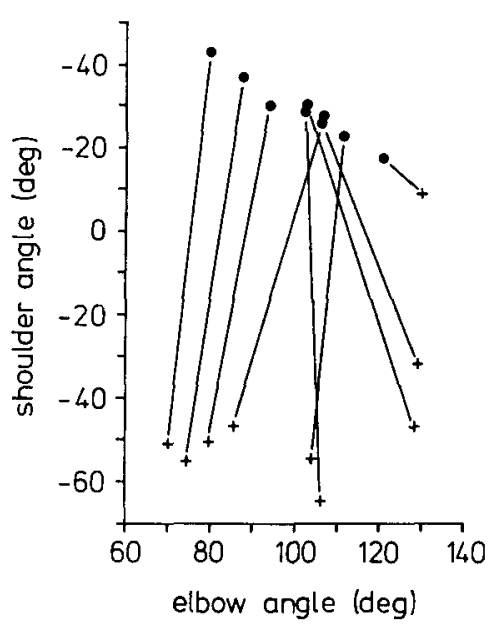

Fig. 6. The dependency of the joint angles at the end of the targeting movement from the angles at the beginning of the movement in the four joint arm. Corresponding starting (crosses) and end positions (circles) are connected by thin lines. For each starting position the arm points to a different point whereas all end positions correspond to the same target point. The latter is the same as that used in Fig. 5. The subject is different

point. This was shown by the following experiment which was performed with 16 subjects. One target point was used starting from 15 different starting positions. For all four joints the standard deviation of the angle values at the target point was measured. The results show a greater standard deviation compared to the case of the three joint arm. According to the results shown in Fig. 5 the arm positions should not follow a random distribution but should be concentrated along the minimum valley. This prediction could be tested by plotting the result in the same coordinate system as was used in Fig. 5a. This result is presented for one subject and one target point in Fig. 5a (filled circles). Each circle represents a position of the arm pointing to the same target. It should be mentioned that a detailed quantitative comparison between experimental and theoretical data is difficult because the form of the cost functions used for the model calculation could only be approximated on a qualitative level. A quantitative analysis will be the subject of a subsequent investigation. Nevertheless, this qualitative comparison shows considerable agreement. Corresponding results are found for the other subjects and other target points. This supports the hypothesis that the minimum cost principle is also applied in the case of the four joint arm, but a rather broader minimum angle range than the exact mathematical minimum is reached. In most subjects significant correlations exist between the end angle and the angle at the beginning of the movement. This is shown for one subject and one target point in Fig. 6. This result shows that the distribution of these data is also dependent upon the history.

\section{Discussion}

The aim of the current investigation was to look for measurable values corresponding to the hypothetical cost functions which were postulated earlier for the control of the redundant arm (Cruse 1986; Cruse and Brüwer 1987). The first question investigated was whether the choice of the arm position depends solely on the values of the joint angles or also on the length of the limbs. Experiments using pointers of different lengths attached to the hand showed that the strategy in choosing the joint angles are independent of the limb length although in these experiments the geometry of the whole arm was changed considerably. However, it should be stressed that this result does not allow any direct conclusions concerning the existence of the cost functions.

Concerning the nature of the cost functions (at least) two assumptions are possible: (a) the cost functions might represent the physiological costs necessary to maintain a given joint angle or $(b)$ they might be completely independent of any kind of physiological costs and merely represent a "computational" value used by the neural system to solve the redundancy problem. Loading the arm during the targeting movement changes the physiological costs. Therefore according to the first assumption different joint angles should be adopted in pointing to a given target position when the elbow joint is loaded. According to the second assumption no differences should occur. The experiments showed ambiguous results when a constant load was applied. However, they showed a clear result supporting hypothesis $(a)$ in those experiments where the joint is loaded by a force which depends on the joint angle. How could this result be explained? The resulting joint positions for a given target point does not depend on the absolute value of the cost functions. According to the minimum cost principle it rather depends on the slope of the cost functions at the angle values under consideration. Regarding the physiological costs, as a first approximation one might assume that adding a constant load shifts the physiological cost function by a constant amount and therefore does not influence the slope of these cost functions (Fig. 7a). This is different when loading takes place by means of a spring. This means adding physiological costs which increase in dependency of the joint angle. Therefore the slope of the physiological cost function would be changed (Fig. 7b). According to the minimum cost principle this would mean that if the hypothetical cost functions corresponded to the physiological costs, no effect should be expected in the first case whereas the joint angle should be shifted in the direction of the force in the second case. This was clearly the case for the elbow joint and, 

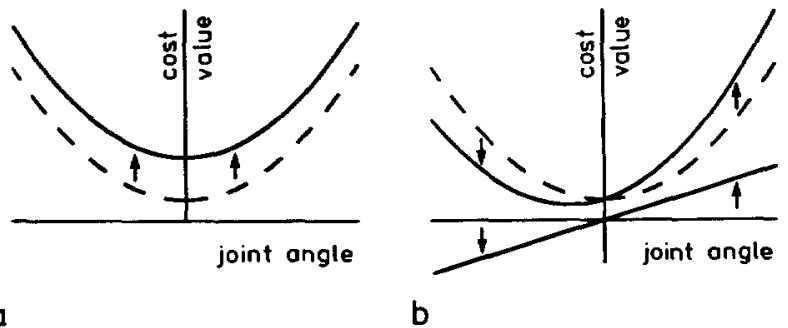

Fig. 7a and b. A schematical representation of the physiological costs when (a) the joint is loaded by an additional constant force and (b) by a force the amount of which depends linearly on the joint angle. In the first case the slope of the cost function at a given angle value is not changed in contrast to the second case. The cost function in the unloaded situation is shown by a dashed line

in the only case which could be investigated, for the shoulder joint. Thus the hypothetical cost functions postulated to control the movement of the human arm seem to be related with the physiological costs necessary to hold the joint in a given position.

Psychophysical experiments revealed $U$-shaped cost functions measuring the comfort of a given joint angle. The "psychophysical" cost functions can be approximated by parabolas the two branches of which in general have different slope values. The position of minimum cost (maximum comfort) for one joint showed very weak dependency on the angles of the other joints. The slopes are on the order of 0.0 to 0.25 . The effect is the opposite to that expected if it arises from the anatomical arrangement of the tendons spanning two neighbouring joints. Thus we assume that a neuronal mechanism is responsible for this coupling effect. For each subject these "psychophysical" cost functions are compared with the hypothetical cost functions. The latter were obtained in targeting experiments as described earlier. They are defined as those functions providing the best fit to the data according to the criterion of the smallest mean square deviation. A qualitative comparison (Fig. 3) shows that there is not a full agreement between both types of cost functions. Nevertheless, the difference is small enough that the results can be considered to support the assumption that the psychophysically measured comfort functions provide a measure for the hypothetical cost functions postulated to explain the targeting movement (Cruse 1986). The result that the hypothetical cost functions depend on the physiological costs further supports the speculation that the physiological costs and the psychophysically measured comfort functions are also related. This assumption agrees with the following result. Measurements of the torque produced in the ankle joint during passive movement of the joint showed a parabola-like dependence (Weiss et al. 1986) which is very similar to the form of our cost functions.

Soechting and Ross (1984) investigated by psychophysical methods which of different possible angle values might be used for a central representation of the position of the arm in the three dimensional space. The shoulder angle considered in our experiments corresponds to one of the two angles which these authors assume to represent the position of the upper arm. The position of the lower arm seems represented by the angle between the vertical and the long axis of the lower arm (Soechting 1982) and, as shown by indirect evidence (Soechting, personal communication) by the elbow joint as was used here.

A successful application of the minimum cost principle to the three joint arm does not necessarily mean that the same principle can also be used concerning the control of the four joint arm which has an additional degree of freedom. Before investigation of the arm with all four joints free to move, two subsystems, the distal and the proximal three joint arm, were investigated separately. Although both subsystems could be sufficiently approximated by application of the minimum cost principle, in the four joint arm several approaches did not provide any adequate result. Nevertheless the results follow the prediction of a model calculation nicely when we assume that not the exact mathematical minimum, but any arm position in a range of nearly minimum costs is considered as a sufficient solution. Up to now the question has remained open which strategies are used to select one of these positions all belonging to the minimum range. Most subjects showed a correlation between the joint angle at the beginning and at the end of the movement. This means that the end position depends on the history. Thus the answer to this question might probably be found by considering not only the static situation at the endpoint of the movement, but also the kinematics of the arm. Investigation of the kinematics of the three joint arm did under some circumstances also show that the end position could depend on the history (Cruse and Brüwer 1987).

In conclusion it can be said that with regard to the three joint arm, the hypothesis of the minimum cost principle is supported by these results. Furthermore, the cost functions which can directly be measured by psychophysical experiments seem to be quite similar to the hypothetical cost functions postulated to explain the control of the movement of the human arm. In addition, the latter seem to depend on the actual physiological costs necessary to hold a given joint position. With regard to the four joint arm, the minimum cost principle seems in principle to be a correct description which is, however, completed by an 
additional strategy which probably has to consider the kinematics.

\section{References}

Cruse $H$ (1986) Constraints for joint angle control of the human arm. Biol Cybern 54:125-132

Cruse $H$ (1989) The control of path and joint angles in a human arm. In: Personnaz L, Dreyfus G (eds) Neural networks from models to applications. IDSET, Paris, pp 71-77

Cruse H, Brüwer $M$ (1987) The human arm as a redundant manipulator: the control of path and joint angles. Biol Cybern 57:137-144

Soechting JF (1982) Does position sense at the elbow reflect a sense of elbow joint angle or one of limb orientation? Brain Res 248:392-395

Soechting JF, Ross B (1984) Psychophysical determination of coordinate representation of human arm orientation. Neuroscience 13:595-604
Stevens SS (1958) Problems and methods of psychophysics. Psychol Bull 55:177-196

Weiss PL, Kearney RE, Hunter IW (1986) Position dependence of ankle joint dynamics. I. Passive mechanics. J Biomech 19:727-735

Received: September 25, 1989

Accepted in revised form: December 4, 1989

Holk Cruse

Fakultät für Biologie

Universität Bielefeld

Postfach 8640

D-4800 Bielefeld 1

Federal Republic of Germany 